\title{
Priority-based Scheduling Algorithm for NOMA-integrated V2X
}

\author{
Ala Din Trabelsi*, Hend Marouane, Faouzi Zarai \\ National School of Electronics and Telecommunications (ENET'COM), NTS'COM Laboratory, Sfax University, 3029, Tunisia
}

\begin{tabular}{l} 
A R T I C L E I N F O \\
\hline Article history: \\
Received: 30 August, 2020 \\
Accepted: 26 November, 2020 \\
Online: 16 December, 2020 \\
\hline Keywords: \\
$5 G$ \\
V2X \\
Scheduling Algorithm \\
Resource allocation \\
PSA
\end{tabular}

\begin{abstract}
A B S T R A C T
The fifth-generation (5G) wireless system was implemented due to the massive connectivity, especially Internet of Things (IoT) including industrial automation, Vehicle to Everything $(V 2 X)$ communications, which is shelter an extensive range of applications services and use cases and Intelligent Transportation Systems (ITS). A cellular Non-Orthogonal Multiple Access (NOMA) based on radio resource allocation schemes need to be carefully designed to serve and support the diversity of applications and services, through increased safety and traffic efficiency usage of connected vehicle and ranging from infotainment services. In this paper, we develop a novel scheduling and resource allocation algorithm for vehicular communications named Priority -based Scheduling Algorithm for NOMA integrated V2X (PSA). The novel scheme could make the limited radio Resource Blocks $(R B)$ to be assigned in a more efficiently manner to the different traffic classes (safety and non-safety traffic) imposed by $V 2 X$ exigency, in order to improve the performances of the system in terms of average throughput, Quality of Service (QoS), Average Blocking Rate $(A B R)$ and to ensure fairness criteria between Vehicular User Equipments (VUEs). The performance of PSA is shown for urban macro-cell and rural macro-cell scenarios and we compared it with the conventional OMA and some recent works for NOMA based allocation.
\end{abstract}

\section{Introduction}

New 5G technologies supporting vehicular communications considered as a promising and vital role in the blueprint of Intelligent Transportation Systems (ITS), assisting to solve the main problems of transport engineering, improves road safety, traffic efficiency and comfort and infotainment services, try to prevent any fostering and dangerous situations investments in the automotive market [1-3].

$\mathrm{V} 2 \mathrm{X}$ connectivity has been developed as a potential concept for $5 \mathrm{G}$ which strive to give communication models that can be supported by vehicles in several application contexts including vehicle-to-vehicle (V2V), vehicle-to-infrastructure/network $(\mathrm{V} 2 \mathrm{I} / \mathrm{N})$ and vehicle-to-pedestrian (V2P) [4].

V2X will include two complementary sets of specifications for direct transmission which are 802.11p and cellular V2X (C-V2X) standards. The first one is proposed as the lower layers of Wireless Access in the Vehicular Environment (WAVE), it is standardized for V2V defined in USA and recognized as Dedicated Short-Range Communications (DSRC). 802.11p is also designed as the lower

${ }^{*}$ Corresponding Author: Ala Din Trabelsi, trabelsi.ala93@gmail.com layers of the ITS-G5 standard in Europe. It is based on OFDM and which is known as the case of fourth generation (4G) technology LTE (Long Term Evolution). However, the adoption of DSRC has been delayed due to its Medium Access Control (MAC) and physical layer designs that have been planned for Wireless Local Area Networks (WLAN) and its poor scalability and the highmobility environments that impose communication challenges, and lack of QoS guarantee. In addition, 802.11p indicates that it's restricted range, reliability and undefined performance especially in urban propagation scenarios when scaling up the number of transmitting VUEs [4].

Cellular-V2X is proposed to innovate and deliver an evolution path to $5 \mathrm{G}$ and it comes to handle the challenges of $802.11 \mathrm{p}$. The main advantage of $\mathrm{C}-\mathrm{V} 2 \mathrm{X}$ that is based on a technology originally optimized for high-speed mobile services, which has been enhanced specifically for safety and automotive applications. C$\mathrm{V} 2 \mathrm{X}$ is outperformed $802.11 \mathrm{p}$ due to modulation and coding enhancement, to support a better communication range, greater capacity, better congestion control in denser environments with lower packet error rate, higher reliability and superior Non Lineof-Sight (NLoS) performance [4]. 
Accordingly, 27 use cases for V2X communications are designed by third-Generation Partnership Project (3GPP) in Release 14 that including two categories of service requirements as safety related applications, like car platooning, autonomous driving, vulnerable road user warning, etc and non-safety related uses cases exemple, dynamic digital map update, mobile hot spot, mobile high data rate entertainment, office, home, etc [5].

More recently, 5G networks anticipate an explosive demand for massive connectivity driven by the ever-increasing number of connected vehicles, Internet of Things (IoT) and the explosive proliferation of mobile Internet, over limited radio resources [6].

The limitation of OMA-based scheme that it has not been efficiently and fully assigning the limited radio resource blocks causing to serious problems as lack of QoS guarantee for NLoS environment. This defiance can be exemplified with the following scenario, consider a single User Equipment (UE) with mediocre channel condition needs to be scheduled for fairness purposes, example this UE has superior priority information or has not been scheduled for a long time. This causes that one of the limited bandwidth resources is only allocated by this UE. In addition, the limitation of the conventional OMA is the number of users that can be served simultaneously. Towards these challenges, 3GPP is motivated to develop new technologies in order to maximizing the achievable throughput. Among various solutions, non-orthogonal multiple access (NOMA) is a very promising access technology. It has been envisioned and recognized as an innovation for $5 \mathrm{G}$ mobile networks to increase the number of links, enlarge the spectral efficiency and to ensure the high data rate necessity. It provides enhancements in terms of high reliability, low latency and communication's range for vehicular environments [7].

Using non-orthogonal radio resource allocation, make NOMA to multiplex more than a few users with different power levels into a same frequency resource and time to transfer information. Not only does NOMA support users with good channel conditions but also it serves both others with poor channel conditions and those operate in the same bandwidth resources [8][9].

To enable V2X communications, radio resource management is a vital specification of the MAC layer of the eNodeB, that aims to optimize the use of the limited availability of the spectrum while meeting the requirements of QoS and insure efficient resources scheduling, resource allocation and interference management. Even in $5 \mathrm{G}$ networks, a resource allocation technique requires to be carefully developed to satisfy different QoS requirements to achieve target throughput and to maximize spectrum efficiency for several V2X traffics and services.

The paper contributions are as following:

- Taking into account traffic classification, such as Safety Traffic (ST) and Non Safety one (NST) are considered

- A resource allocation algorithm called Priority -based Scheduling Algorithm for NOMA integrated V2X (PSA) is proposed.

- Adding a scheduling priority which is determined according to the channel condition for each VUE, users with higher priority will be selected for NOMA sharing.
- Due to the diversity and multitude of the propagation scenarios, various simulations are presented for performance evaluation for rural macro-cell and urban macro- cell, to demonstrate the efficiency and the applicability of the proposed PSA algorithm for any propagation scenarios.

- Validate the proposed PSA algorithm with several Matlab simulations showing the aggregate throughput, the fairness index and the ABR among different VUE traffic classes.

- Compare the proposed algorithm with the conventional OMA

- Evaluate the PSA algorithm with some recent works for NOMA based allocation.

This paper is presented as follows we study same proposed method as related works in section 2. We detail our approach in section 3 . In the section 4 and 5 we portray its different steps. We evaluate and analyze the performances of the developed scheduler in section 6. Finally, in Section 7, we determine our work by conclusion and perspective as future work.

\section{Related Work}

The radio resources management task to enable V2X communications has been investigated in several research works such as:

In [10], the authors developed a novel resource allocation algorithm to maximize the number of scheduled V2N communications while satisfying the required latency for the several priority classes of the V2V links (two class of priority are defined, where the messages in the class 1 are more critical and aware to the latency comparing to messages in the second one). They perform the power allocation for potential resource reusing. Then, they selected the finest reusing pattern by employing the Hungarian scheme. However, this method does not take NOMA systems in consideration and the performance evaluations focused only to present the average latency.

In [11], the authors studied the resource allocation process when the periodic messages are not all at the same size in cellular V2X networks. They addressed an optimization problem for maximizing number of vehicles that can be allocated simultaneously without interfering each other. Then, they provided the same algorithm to solve optimization problem then evaluate its performance by increasing number of vehicles when a trade-off between range and density of vehicle is guaranteed. However, NOMA scenarios issue was usually ignored in design of the resource allocation algorithm.

In [12], the authors developed a novel resource allocation scheme for Power domain based a cooperative multi-cell network by using the cellular mode. This algorithm aims to improve the spectral efficiency of the system and achieves the desired QoS. They assume a communication scenario for two pairs of users sharing the same sub-carrier to transmit their information, where the primary users characterized by delay-sensitive communication that requires stringent QoS exigency as well as the minimum transmission rate, share the same radio RB with the secondary users which support non-delay sensitive or elastic traffic and exploit the physical resources of the network in a best-effort manner. Successive convex approximation (SCA) method is the 
best solution to solve general optimization issues, they adopt the successive convex approximation (SCA). Furthermore, they investigate the computational complexity of the designed approach and they compare our work with existing transmission methods based on Orthogonal Frequency Multiple Access (OFDMA). However, this approach does not hit the V2X links requirements.

Some recent research has begun to explore efficient energy NOMA design were given in [13-16]. They presented a general overview of NOMA techniques and seek to optimize the power allocation problem in order to improve Energy Efficiency (EE). Then, they proposed a low complexity power allocation approach. However, those algorithms do not cover V2X links, traffic classifications and heterogeneous QoS requirements for each VUE link.

In [17], the authors focus on the problem of clustering users in groups of different size, and assigning RB to each cluster based on Power-Domain (PD-NOMA) scheme. Clustering and RB allocation are performed as stated by Proportional Fairness (PF) technique with the main objective that obtaining high cell throughput, low complexity and guaranteeing NOMA relevance. The performance evaluation of the clustering algorithm proposed Relevant Clustering (RC) compared with two other algorithms named Ordered Clustering (OC) and Disparity Clustering (DC). However, traffic classifications such safety information for the $\mathrm{V} 2 \mathrm{X}$ links is not considered in this algorithm.

In [18], the authors improved proportional fair scheduling scheme for NOMA system, called a spectrum resource and power allocation algorithm with Adaptive Proportional Fair (APF) user pairing with low complexity. This algorithm aims to maximize system throughput and considers the fairness among UE by selecting the user pairing with higher priority expressed by the channel gain. For the optimizing the power allocation, they explore different power allocation schemes which focusing on the proportional rate constraint so as to ensure the best throughput performance. The limitation of this approach that does not considering V2X traffic classifications.

In [19], the authors have addressed and investigated the power allocation problem in downlink Multiple Carriers Non-Orthogonal Multiple Access (MC-NOMA) systems for maximizing Weighted Sum Rate (WSR) by considering imperfect successive interference cancellation, in order to present an iterative power allocation with low-complexity algorithm. Moreover, they have presented a joint user scheduling and power allocation scheme for the MC-NOMA systems. The proposed methods can achieve an optimal WSR performance and user fairness improvement compared to OMA and other existing schemes. However, the system model only considered each sub- channel can be multiplexed by two users no user priority and service classification are treated.

In [20], the authors investigated the benefits of $5 \mathrm{G}$ technologies based on NOMA to support massive connections services to group and assign the required sub-channel of the internet of things equipment. To show how the quality of experience requirements of IoT links affect the needed number of physical RBs in real time, they developed a novel method to improve spectrum distribution and to control IoT links mobility by using Non-Orthogonal Multiple Access technique for autonomous vehicles. The designed solution aims to maximize throughput, to contribute QoS improvement and exploiting k-means grouping to reply to the dynamics of the system and minimize the complexity of rearranging NOMA clusters. The regrouping of IoT connections according to distance while considering the maximum supported devices by group. Therefore, they determined the requisite set of $\mathrm{RB}$ for each group of UEs based on the QoS requirement. However, IoT equipments are clustered without considering their type of traffic.

In [21], the authors is introduced an over view on optimization problems of scheduling and NOMA approaches. They planned a novel scheduling and resource allocation scheme based on NOMA-enhanced cellular network for device-to-device (D2D) communications called Tree-based Search power allocation Algorithm (TSA). This scheme is compared and evaluated with several statistic models for user scheduling algorithms, users selection problems for the D2D communications underlying cellular network based on NOMA technology and power allocation strategies such as FRPA (FRactional Power Allocation) and FIPA (FIxed Power Allocation algorithm) where their mean objectives are mitigating computational complexity of the algorithm, increase network capacity, guarantying the QoS and maintaining a better system throughput. The simulation results clearly demonstrate the superiority of TSA compared with traditional algorithms. However, the proposed algorithm does not consider V2X links and traffic classification (ST and NST).

In [22], the authors designed a new method entitled proportional-fairness based user pairing and power allocation algorithm for NOMA uplink transmission. This method is introduced to serve two scenarios: the first one is when user equipments are only distributed in the coverage area of the cell and the second one which is the more complex that is when the interference users are distributed outside of the cell. For scenario one, to optimize the problem they decoupled the User Pairing (UP) part and Power Allocation (PA) part. In the power allocation part, they classify the pairs of users by considering their channel condition and SINR constraint and they developed different power allocation techniques. While in the user pairing part, they designed a probability-based tabu search user-pairing algorithm (PTS). For scenario two, they carried out a prediction-based particle swarm optimization (PBPSO) power allocation algorithm with a low computational complexity to obtain a good proportional fairness performance under the constraints of outage probability. Then, they combine PBPSO and PTS to achieve high system performance in terms of average throughput and outage probability. However, this algorithm assigns the RB for the pairs of users independently of their type of traffic class.

In [23], the authors investigated the global throughput maximization for two UE with timeliness transmission over fading channels for partial and full Channel State Information at the Transmitter (CSIT). This approach supports certain level of fairness and the minimum average throughput constraint. In the full case of CSIT case, the dynamic resource allocation method was optimally determined by using lagrangian dual decay, while in the partial CSIT one, the power allocation technique was proposed focused on investigative results. However, the limitation of this algorithm that does not takes into account the demanded QoS requirements for users and the traffics classifications. The simulation results are investigated with limited parameters setting. 
In addition, the required bandwidth to enable $\mathrm{ST} V 2 \mathrm{X}$ services in NOMA has not been studied.

In [24], the authors are introduced an over view on the optimization scheduler problem joined with NOMA techniques. They developed and simulated some statistic models for user scheduling strategies and power allocation approaches which aim to achieve the different objectives as minimizing the computational complexity of the algorithm, satisfy the required QoS and obtaining a better system performance in terms of average sum rate and equity criteria. Also, they select Proportional Fairness (PF) and Round-Robin (RR) as a shared model of scheduling and NOMA techniques, to evaluate the optimization problem of power allocation for combined NOMA and round-robin scheduling scheme and proposing an optimization strategy for joint NOMA algorithms and Proportional Fairness scheduling which assure the required QoS. However, the proposed scheme doesn't reflect on the V2X link environments and traffic classifications.

The table 1 illustrates all the mentioned algorithms above. All related work mentioned above doesn't take charge the traffic classifications while seeking the throughput performance gain. Nevertheless, the benefits of V2X ST class at the road cannot be exploited successfully by these algorithms. Despite the existing literature, there is a lack of priority constraint-based scheduling scheme that marked the transmission ranking to each connection but it restricted just by the Channel Quality Indicator (CQI). In addition, diverse algorithms presented above didn't balance cell throughput, equity index and take the system-level QoS for V2X links, new solutions must be provided.

\section{System model}

The used input and variable parameters are given below.

\subsection{System Model}

In order to evaluate our proposal work, we consider NOMA scheme consists of a single base station and $\mathrm{N}$ connected VUEs denoted by $\mathrm{I}=\{1, \ldots . \mathrm{j}, \ldots, \mathrm{N}\}$. We suppose that the number of available RBs in the cell is $\mathrm{M}$ where the resource blocks set $\mathrm{U}=$ $\{1, \ldots i, \ldots, M\}$. To assure the required capacity of the $N$ vehicular connections, we suppose that PD-NOMA (Power-Domain NOMA) technique linking by the base station. NOMA is a scheme used to appreciate multiple accesses by utilizing power domain in a spectrally resourceful way and can schedule multiple vehicular user equipments in the same radio resource. This can be maintained by assigning dissimilar power levels to VUEs. Moreover, NOMA scheme is expected to allocate more power level to the VUEs with poor channel qualities. Denote the power of $\mathrm{VUE}_{\mathrm{j}}$ on subcarrier $\mathrm{k}$ by $\mathrm{P}_{\mathrm{j}, \mathrm{k}}$, satisfying $\sum_{\mathrm{j} \in \mathrm{N}} \mathrm{P}_{\mathrm{j}, \mathrm{k}} \leq \mathrm{P}_{\mathrm{j}}^{\text {max }}$, where $P_{j}^{\max }=P$ is the maximum power constraint allocated for $\mathrm{VUE}_{\mathrm{j}}$. Therefore, we assume that $\mathrm{N} \geq 2 \mathrm{M}$ for dense environment and each vehicular user assign to one RB in the same Transmission Time Interval (TTI) while the entire other not scheduled vehicular users are not allocated. In addition, we assume that the distribution and the position of links are supposed random in the coverage area.

To maintain the improvements of $\mathrm{V} 2 \mathrm{X}$ link, radio resource allocation and Transmitter -Receiver selection are managed by the eNodeB in each TTI.

Table 1: Comparison between different algorithms studied above

\begin{tabular}{|c|c|c|c|c|c|c|}
\hline \multirow[b]{2}{*}{ Ref } & \multirow{2}{*}{$\begin{array}{l}\text { NOMA } \\
\text { systems }\end{array}$} & \multicolumn{2}{|c|}{ Throughput optimization } & \multirow{2}{*}{ Qos satisfaction } & \multirow{2}{*}{$\begin{array}{c}\text { Services } \\
\text { classifications ST } \\
\text { / NST }\end{array}$} & \multirow{2}{*}{ Fairness } \\
\hline & & Average & Instantaneous & & & \\
\hline [10] & $\sqrt{ }$ & $\sqrt{ }$ & & & & $\sqrt{ }$ \\
\hline [11] & $\sqrt{ }$ & $\sqrt{ }$ & & & & \\
\hline [12] & $\sqrt{ }$ & $\sqrt{ }$ & & $\sqrt{ }$ & & \\
\hline [13] & $\sqrt{ }$ & $\sqrt{ }$ & & & & \\
\hline [14] & $\sqrt{ }$ & $\sqrt{ }$ & & & & \\
\hline [15] & $\sqrt{ }$ & $\sqrt{ }$ & & & & \\
\hline [16] & $\sqrt{ }$ & $\sqrt{ }$ & & & & $\sqrt{ }$ \\
\hline [17] & $\sqrt{ }$ & $\sqrt{ }$ & & & & \\
\hline [18] & & $\sqrt{ }$ & & & & \\
\hline [19] & $\sqrt{ }$ & $\sqrt{ }$ & & $\sqrt{ }$ & & \\
\hline [20] & $\sqrt{ }$ & $\sqrt{ }$ & & & & \\
\hline [21] & $\sqrt{ }$ & $\sqrt{ }$ & $\sqrt{ }$ & $\sqrt{ }$ & & \\
\hline [22] & $\sqrt{ }$ & & $\sqrt{ }$ & & & $\sqrt{ }$ \\
\hline [23] & $\sqrt{ }$ & $\sqrt{ }$ & & $\sqrt{ }$ & & $\sqrt{ }$ \\
\hline [24] & & & $\sqrt{ }$ & & Only ST & \\
\hline
\end{tabular}

Table 2: Notations

\begin{tabular}{|l|l|}
\hline \multicolumn{1}{|c|}{ Symbol } & \multicolumn{1}{c|}{ Description } \\
\hline $\mathrm{N}$ & Connected VUEs. \\
\hline $\mathrm{I}$ & Set of vehicular uses equipments. \\
\hline $\mathrm{M}$ & Available RBs. \\
\hline $\mathrm{U}$ & Set of resources blocks \\
\hline
\end{tabular}




\begin{tabular}{|l|l|}
\hline $\mathrm{P}_{\mathrm{ST}}, \mathrm{P}_{\mathrm{MT}}, \mathrm{P}_{\mathrm{IT}}$ & Percentage of safety, management and infotainment class of traffic. \\
\hline $\mathrm{N}_{\mathrm{ST}}, \mathrm{N}_{\mathrm{MT}}, \mathrm{N}_{\mathrm{IT}}$ & Number of active VUEs for each class of traffic. \\
\hline$\varphi, \psi$ & Set of safety and non safety VUEs. \\
\hline $\mathrm{N}_{\mathrm{NST}}=\mathrm{N}_{\mathrm{MT}}+\mathrm{N}_{\mathrm{IT}}$ & Number of NST VUEs. \\
\hline$\dot{\mathrm{C}}, \dot{\mathrm{R}}$ & Set of ST and NST VUEs candidates that can be served to different resource. \\
\hline P $_{\text {factor }}$ & The power level for VUE. \\
\hline H_ST & Channel coefficient of safety VUE. \\
\hline H_NST & Channel coefficient of non-safety VUE. \\
\hline thr_min_safety & Minimum throughput for safety traffic requirement. \\
\hline H_safety & Matrix contains the channel coefficient of safety VUEs. \\
\hline H_nonsafety & Matrix contains the channel coefficient of non- safety VUEs. \\
\hline
\end{tabular}

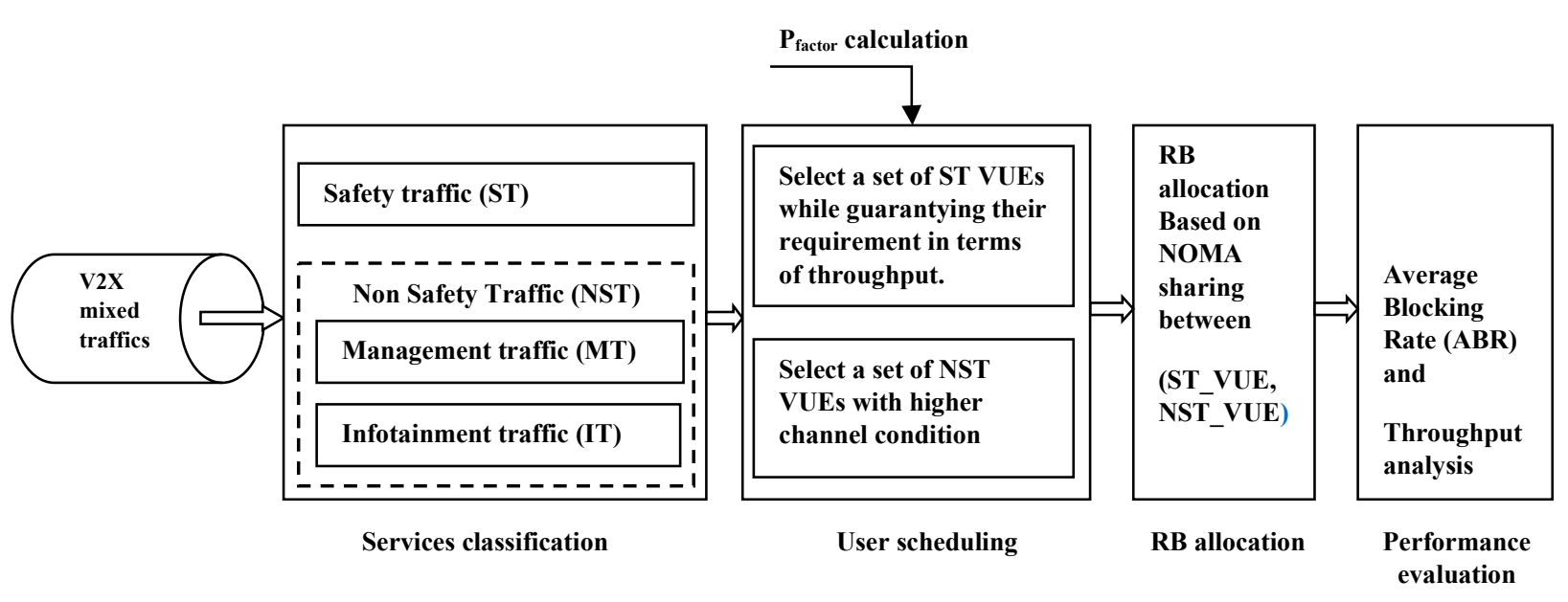

Figure 1: PSA system model

Using NOMA system, the eNodeB superimposes the message waveforms with different assigned power level for its selected users. At the Rx, successive interference cancellation (SIC) schemes are employed to cancel multi-user interferences and each user detects its own signal. The receivers with higher channel gain apply SIC for signal decoding. Therefore, those the modulated symbols are subtracted and removed from the united data until the associated UE obtains the desired message [6].E.g., consider two Tx: $V_{U} E_{j 1}$ and $V E_{j 2}$ occupying the $R_{i}$ in the current TTI transmitting to receiver $\operatorname{VUE}_{m}$ satisfying $\mathrm{P}_{\mathrm{j} 1, \mathrm{k}}^{(\mathrm{i})}\left|\mathrm{H}_{\mathrm{j} 1, \mathrm{~m}, \mathrm{k})}^{(\mathrm{i})}\right|^{2}<$ $\mathrm{P}_{\mathrm{j} 2, \mathrm{k}}^{(\mathrm{i})}\left|\mathrm{H}_{\mathrm{j} 2, \mathrm{~m}, \mathrm{k}}^{(\mathrm{i})}\right|^{2}$, where $\mathrm{P}_{\mathrm{jn}, \mathrm{k}}^{(\mathrm{i})}$ representing the transmit power of the transmitter $\mathrm{VUE}_{\mathrm{jn}}, \mathrm{n} \in\{1,2\} . \mathrm{H}_{\mathrm{j} 2, \mathrm{~m}, \mathrm{k}}^{(\mathrm{i})}$ presentes the channel coefficient of the subchannel $\mathrm{k}$ which allocated for the vehicular user $V U E_{j n}$ in the $R_{i}$. The receiver $V_{U E}$ deals the signal of the great channel condition $\mathrm{VUE}_{\mathrm{j} 2}$ as a noise, first, detects and remodels the signal of the inferior channel gain of $\mathrm{VUE}_{j 1}$, then, exploits the SIC technique to cancel or withdraw the signal of $\mathrm{VUE}_{\mathrm{j} 1}$ which has the mediocre channel condition to get signal of $\mathrm{VUE}_{\mathrm{j} 2}$.

Generally, the signal $\mathrm{x}_{\mathrm{k}}$, transmitted by the eNodeB at a certain sub-band $\mathrm{k}$ is a simple power multiplexing of the symbols generated by the scheduled users.

$$
\mathrm{x}_{\mathrm{k}}=\sum_{\mathrm{j} \in \mathrm{N}_{\mathrm{m}}} \mathrm{x}_{\mathrm{j}, \mathrm{m}, \mathrm{k}}=\sum_{\mathrm{j} \in \mathrm{N}_{\mathrm{m}}} \sqrt{\mathrm{P}_{\mathrm{j}, \mathrm{k}}^{(\mathrm{i})}} \mathrm{s}_{\mathrm{j}}^{(\mathrm{i})}
$$

Denotes $\mathrm{N}_{\mathrm{m}}=\left\{1 \leq \mathrm{j} \leq \mathrm{N} \mid \max \left(\operatorname{SINR}_{\mathrm{j}, \mathrm{m}}^{(\mathrm{i})}\right)\right\}$ : presents the set of VUEs inside receiver vehicular UE $\mathrm{m}$ 's communication range of interest. $P_{j, k}^{(i)}$ is the power allocated to user $j$ at sub-band $k$ and $S_{j}^{(i)}$ indicates the transmitted signal of $\mathrm{Tx} \mathrm{VUE}_{\mathrm{j}}$.

The received signal vector corresponding to the time slot $\mathrm{i}$ can be defined by:

$$
\begin{aligned}
Y_{m, k}^{(i)} & =H_{j, m, k}^{(i)} x_{j, m, k}+n_{m}^{(i)} \\
& =\sum_{j \in N_{m}} \gamma_{j}^{(i)} \cdot \mu_{j, k}^{(i)} \sqrt{P_{j, k}^{(i)}} H_{j, m, k}^{(i)} S_{j}^{(i)}+n_{m}^{(i)}
\end{aligned}
$$

where:

$\gamma_{j}^{(i)}$ : Binary variable denotes that $\mathrm{RB}_{\mathrm{i}}$ is assigned by the $\mathrm{BS}$ to $\mathrm{VUE}_{\mathrm{j}}$.

$\mu_{\mathrm{j}, \mathrm{k}}^{(\mathrm{i})}$ : Binary variable indicates that subchannel $\mathrm{k}$ is assigned by the eNodeB to $\mathrm{VUE}_{\mathrm{j}}$.

$\mathrm{H}_{\mathrm{j}, \mathrm{m}, \mathrm{k}}^{(\mathrm{i})}$ : presents the channel coefficient of subchannel $\mathrm{k}$ between $\mathrm{Tx}_{\mathrm{j}}$ and $\mathrm{Rx}_{\mathrm{m}}$ in $\mathrm{RB}_{\mathrm{i}}$.

$\mathrm{n}_{\mathrm{m}}^{(\mathrm{i})}: \mathrm{CN} \sim\left(0, \sigma^{2}\right)$ is the additive white Gaussian noise (AWGN) for reciever $\mathrm{VUE}_{\mathrm{m}}$, and $\sigma^{2}$ is the noise variance. Furthermore, the channel gain can be defined as: 


$$
H_{j, m}^{(i)}=G \cdot h_{j, m, k}^{(i)} \cdot \beta \cdot\left(d_{j, m}^{(i)}\right)^{-\alpha}
$$

Denotes:

G: Constant power gain factor implied by the amplifier and antenna.

$\mathrm{h}_{\mathrm{j}, \mathrm{m}, \mathrm{k}}^{(\mathrm{i})}$ : Complex Gaussian variable $\mathrm{CN}(0,1)$ indicates the rayleigh fading.

$\beta$ : Shadowing fading.

$\mathrm{d}_{\mathrm{j}, \mathrm{m}}^{(\mathrm{i})}$ : Distance between $\mathrm{VUE}_{\mathrm{j}}$ and $\mathrm{VUE}_{\mathrm{m}}$.

$\alpha$ : Path loss exponent.

\subsection{Propagation Scenario}

Many covered propagation scenarios are detailed in [25] and [26]. It is indispensable to extend such modeling and measurement efforts to various environments that are also very important for V2X communications, mainly when safety applications are supported. Moreover, modeling a radio channel has been one of the most challenges in the mobile radio system design, and it is based on measurements specifically made for an intended communication system. Propagation models are the base for channel modeling, as they try to describe the way a radio signal changes during its travel distances from the Tx to the Rx and to mitigate interference. Also, radio propagation has a significant impact on the performance of cellular mobile radio systems due to increased data rate, bandwidth, mobility and QoS.

Then more, the trajectory between the Tx and the R can differ from a simple Line Of Sight (LOS) to a line that is obstructed by mountains, buildings, or foliage, named Non line of sight (NLOS).

The main characteristics of the radio channel - such as path loss, fading and time-delay spread and the parameterization vary from each scenario to an other one similar environments.

The macro-cell propagation can be divided into two classes as urban macro-cell propagation and rural macro-cell one. As the surrounding building heights, the density of obstacles and the number of users is very high in urban area, the cellular network is more robust and complicated where one macrocell antenna serves many microcells and picocells to ensure a better service compared to rural area because of the presence of less buildings and obstacles in the area [26].

For our work, we focus on rural macro-cell and urban macrocell senarios with vehicles travelling on multiple lanes and on random position in the cell, including other pedestrians. Since we are considering the network-controlled allocation under independent Rayleigh fading channel and the line of sight (LOS) propagation properties of the vehicular networks, without loss of generality, we only incorporate the effect of the distance dependent path loss between the eNodeB and each VUE into analysis. Therfore, Path-loss models play an important role in the design of cellular systems to specify key system parameters such as antenna heights, frequency, transmission power and so on.

According to [26], The path-loss models are parameterized and controlled using results from a wide-ranging set of measurement campaigns, and they are extensively used and accepted.
Path loss (PL) is a measure of the average radio frequency attenuation suffered by a transmitted signal when it reaches the receiver, after traversing a path of several wavelengths [25]. It is defined by:

$$
\mathrm{PL}=\alpha * \log 10(\mathrm{~d}[\mathrm{~m}])+\mathrm{B}+\mathrm{C} * \log 10(\mathrm{fc}[\mathrm{Ghz}] / 5)
$$

Were, the fitting parameter $\alpha$ includes the path-loss exponent, $\mathrm{B}$ is the intercept, $\mathrm{C}$ describes the path loss frequency dependence and $\mathrm{fc}$ is the center frequency.

However, the path loss frequency dependencies model used in our scenarios are defined below:

$$
\begin{aligned}
& \mathrm{PL}_{\mathrm{Rura}}[\mathrm{dB}]=40 * \log 10(\mathrm{~d}[\mathrm{~m}])+10.5-18.5 * \log 10\left(\mathrm{~h}_{\mathrm{BS}}[\mathrm{m}]\right)- \\
& 18.5 \log 10\left(\mathrm{~h}_{\mathrm{MS}}[\mathrm{m}]\right)+1.5 * \log 10(\mathrm{fc} * 1 \mathrm{e}-9 / 5) \\
& \mathrm{PL}_{\mathrm{Urban}}[\mathrm{dB}]=40 * \log 10(\mathrm{~d}[\mathrm{~m}])+13.47-14 * \log 10\left(\mathrm{~h}_{\mathrm{BS}}[\mathrm{m}]\right)- \\
& 14 * \log 10\left(\mathrm{~h}_{\mathrm{MS}}[\mathrm{m}]\right)+6 * \log 10\left(\mathrm{fc}^{*} 1 \mathrm{e}-9 / 5\right)
\end{aligned}
$$

where, $h_{B S}$ and $h_{M S}$ are the antenna heights at the base station and the mobile station respectively. $d$ denotes the distance between the Tx and the Rx.

\section{Proposed Algorithm}

This proposed technique aims achieve a higher cell throughput, consider the heterogeneity of VUEs channel gains and the equity criteria. This algorithm trys to increase the number of served safety V2X links in the cell while a minimum requirement must be guaranteed on terms of throughput and to maximize the average data rate of the NST VUEs.

Our proposed algorithm named Priority -based Scheduling Algorithm for NOMA integrated V2X (PSA), can be considered as an assignment solution that aims to obtain an efficient and optimal spectrum allocation for VUE. Firstly, we begin by services classification, also, the incoming links of the VUE are classifyed according to their types of services as: safety traffic and non safety traffic. The NST is divited into two type of services as Management Traffic (MT) and Infotainment Traffic (IT).

Then, we assign the physical resources blocks to the pairs VUEs which can occupy the same spectrum by taking into account the channel condition of the transmitters on each RB. The designed approach enhances the equity index in the processus of resource allocation among diverse VUEs class of services. In fact, if the selected VUE has already assigned to an RB during the current TTI, this one will have't another radio resource block in the same TTI while all the selected users are not served. This, at the same time, raises the spectral efficiency and the capacity of the system inside the coverage area, and ensures a better compromise between the average throughput and the fairness index.

Once, the RB allocation is performed, a power control process is used to treat the interference and minimize the binary error rate by calculating per each resource block the $\mathrm{P}_{\text {factor }}$ that allows the safety user to obtain the needed throughput as well as maximizing throughput of the non-safety user, also, each VUE can attain on the allocated RB without minimizing its achieved throughput. The non-scheduled safety VUEs in the present TTI will be selected with the superior priority to be served for the subsequently TTI. The scheduling algorithm of our system is shown in Figure 1. 
The mean objective of our proposed algorithm PSA is maximizing: the cell throughput, the number of served VUEs, the number of served safety VUEs, the sum rate of non-safety communications. We try to minimize the interference inter VUEs using the same RB by imposing a power constraint for different vehicular UEs.

\section{Description of the Proposed Algorithm}

In this part, we present more details for all steps of our proposed strategy.

\subsection{Step 1: Service Classification}

NOMA resource allocation schemes need to be designed to serve the ever-increasing number of vehicles that are connected for different services, such as safety features and passengers multimedia amenities.

V2X services are a real time traffics and arrive with strict exigency on throughput, reliability and latency. Also, we consider two types of service classes as Safety Traffic (ST) that imposes timeliness requirements, network scalability, and stringent reliability and refers to the case when the transmit signals need high QoS and requires to be selected almost momentarily in order to minimize road accidents, such as traffic condition warning, curve speed warning, vulnerable road user warning and emergency vehicle warning [27].

The NST (Non Safety traffic) included two categories of services: MT and IT. The Management traffic required to be scheduled with most importance thanks to their significance which aims to enhance traffic flow by minimizing traffic congestion and travel time, examples traffic information and recommended, intersection management, regulatory speed limit and automated parking system [28].

Infotainment Traffics are characterized by the delay tolerance and provides convenience applications and comfort for VUEs to improve their driving experience, exemples media downloading, vehicle software update and data provisioning [28].

The idea of the first step is to sort the arriving vehicular links according to their type of service that are: ST, MT and IT.

\subsection{Step 2: User Scheduling}

Scheduler functionality is to allocate the RBs to the UEs according to their requested requirements and their radio conditions at every TTI. So, the available resources blocs are very limited and it has to be allocated with an optimal manner that highest cell throughput and equity index are ensured among all VUEs, applications or service classes.

The system comprises two categories of V2X communications. Let $\varphi=\left\{1 \ldots \mathrm{j}, \ldots . \mathrm{N}_{\mathrm{ST}}\right\}$ the set of Vehicular users with Safety Traffic (VST) and $\psi=\left\{1 \ldots j^{\prime}, \ldots \mathrm{N}_{\mathrm{NST}}\right\}$ the set of VUEs with Non Safety Traffics (VNST), where $\{\varphi, \psi\} \in\{I\}$.

Moreover, the purpose of this step is: firstly, to select a set of VST by $C \in\{\varphi\}$ while guarantying their requirement in terms of throughput. The idea is to search the combination $\left(V_{S S T}, R_{i}\right)$ where $1 \leq j \leq N_{S T}$ and $1 \leq i \leq M$, with the highest metric on the $R B_{i}$. This metric expresses the channel gain between the $\operatorname{VST}_{\mathrm{j}}, \mathrm{j} \in \mathrm{N}_{\mathrm{ST}}$ and the receiver $m$ on subcarrier $k$, denotes by $\left|\mathrm{H}_{\mathrm{j}, \mathrm{m}, \mathrm{k})}^{(\mathrm{i})}\right|^{2}$, which includes both small-scale Rayleigh fading and large-scale path loss.

Secondly, to select a set of VNST by Ŕ $\{\{\}$ that will be shared the same RB $\mathrm{R}_{\mathrm{i}}$ with VST. The selection of VNST is based on the higher channel condition expressed by the estimated SINR which corresponds to the CQI (Channel Quality Indicator) index sent by the VNST to the eNodeB.

Also, priority users scheduling considered as an important tasks to enhance the network performances in terms of aggregate sum rate and equity index before the resource allocation.

\subsection{Step 3: RBs Allocation}

The third step is designed based on NOMA to maximize the aggregate throughput of the system by assigned the limited physical RB to pair of VUEs (VSTj, VNSTj') selected in the above step. Exploiting the knowledge on vehicle positions and propagation characteristics at the eNodeB, this latter allocate the resources to guarantee the required quality of service and maximize the network capacity. Moreover, the proposed RB allocation method is divided on two processes. Starting with the $\mathrm{RB}$ allocation for ST communications which aims to allocate the candidate $C \in\{\varphi\}$ safety users to different resource blocs and set a minimum requirement must be guaranteed for those. Then, the $\mathrm{RB}$ allocation for VNST candidate $\mathrm{R} \in\{\psi\}$

The proposed scheme offered superior priority to the safety links than others type of traffics because they can't tolerate delay and must be scheduled and served immediately without forgetting to taking into considiration the equity index. Moreover, in this step the scheduler checks if each VST has the minimum requirement in terms of throughput. In fact, if the VUE is't satisfied, for the scheduler, this vehicular user is reselected to transmit for the next TTI. This allows us to maximize the served VUEs number by trying to give each one his requested requirement. The algorithm for RB allocation is summarized as following.

\footnotetext{
Algorithm 1: RB allocation

\{Initialization\};

// select the set B safety VUE candidates can be served to different resource blocs;

Check each line of the matrix H_safety and calculate the matrix rb_acceptable_safety : if a single allocation is acceptable that is used (where an allocation can be used is ' 1 ' or can't be used is ' 0 '); at_least_one_allocated = true;

While $i=1$ to $N$ at_least_one_allocated do at_least_one_allocated $=\overline{\text { false; }}$

For $i=1$ to all the vehicle of safety traffic do if $\operatorname{sum}\left(r b \_a c c e p t a b l e \_s a f e t y(i,:)\right)==1$ then Find the best VUE candidate to be served at the rb_to_allocate; Select the best VUE candidate to be served at the rb_to_allocate;
} 


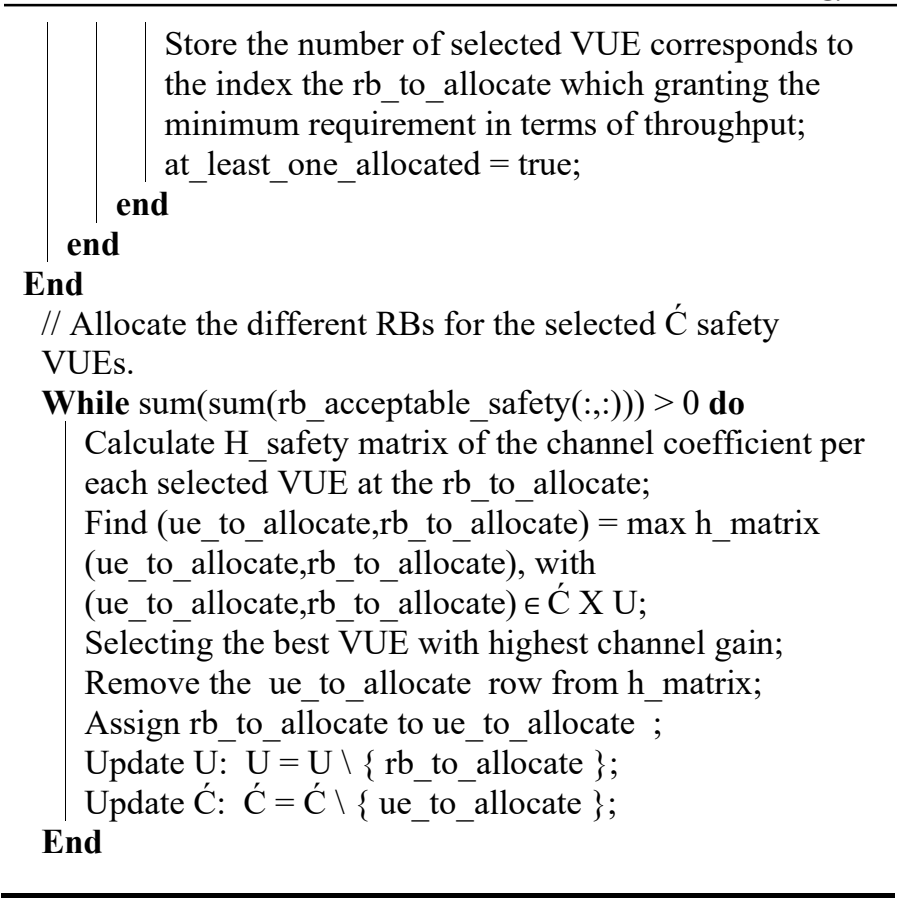

We define an indicator defined by a variable $s_{j}^{i}$, $(i \in M, j \in N)$ which indicates if the $i^{\text {th }} \mathrm{RB}$ is assigned to $\mathrm{j}^{\text {th }} \mathrm{VUE}$ or not.

$\mathrm{S}_{\mathrm{j}}^{\mathrm{i}}=\left\{\begin{array}{ll}1 & \text { if } \mathrm{RB}_{\mathrm{i}} \text { is used by } \mathrm{VUE}_{\mathrm{j}} \\ 0 & \text { otherwise }\end{array}\right\}$

This step is designed to maximize non safety vehicular users throughput (for both management and infotainment VUEs) by prioritizing the users with good channel condition and not to starve others with bad channels condition.

Specifically, the SINR at the receiver $\mathrm{Rx}$ with the highest channel gain which can cancel the interference is computed as:

$$
\operatorname{SINR}_{\mathrm{j}, \mathrm{k}}=\frac{\mathrm{s}_{\mathrm{j}}^{(\mathrm{i})} \mathrm{P}_{\text {factor }}^{(\mathrm{i})} \mathrm{P} \rho_{\mathrm{j}, \mathrm{m}, \mathrm{k}}^{(\mathrm{i})}}{1+\sum_{\substack{\mathrm{l} \in \mathrm{L} n \\ \mathrm{l} \neq \mathrm{j}}}\left(1-\mathrm{P}_{\text {Factor }}^{(\mathrm{i})}\right) \mathrm{P} \rho_{\mathrm{l}, \mathrm{m}, \mathrm{k}}^{(\mathrm{i})}}
$$

where Ln represents the subset of the interfered VUEs when the receiver $m$ exploiting the resource block for the transmission.

\subsection{Step 4: Performance Evaluation}

To collect the benefits of the output of our proposed scheme, certain performance evaluations are need to be addressed such as cell throughput and average blocking rate.

Algorithm 2 briefly describes this process.

Due to the unavailability of radio resources, ABR is a typical constraint for VUE with safety traffic (VST) in PSA which aims to maximize served VST thanks to their high priority, great QoS requirement and due to better road utilization and surroundings. The average blocking rate is calculated as following:

$$
\mathrm{ABR}=\frac{\text { Number of blocked transmitters }}{\text { Total number of VST }}
$$

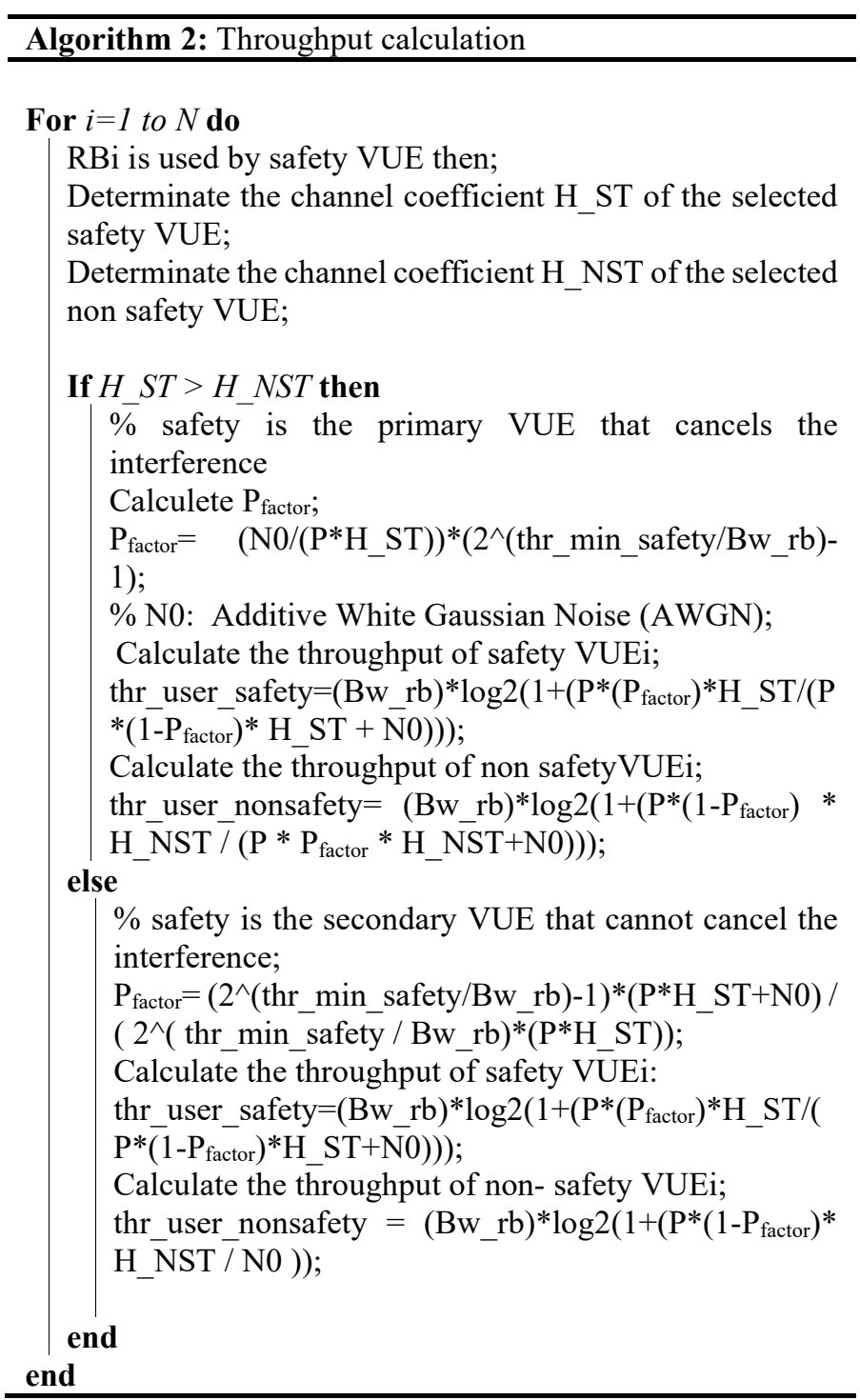

In this step we calculate per each resource block the Pfactor that allows the safety VUE to obtain the needed throughput and to maximize the sum rate of non-safety VUE at the same time. Indeed, the objective is to take into account power split between the two vehicular users which share the same RB to minimize the interference.

The achievable data rate obtained from $\mathrm{VUE}_{\mathrm{j}}$ over sub-channel $\mathrm{k}$ at the $\mathrm{RB}_{\mathrm{i}}$ can be mathematically formulated as:

$$
\begin{aligned}
& \mathrm{R}_{\mathrm{j}, \mathrm{m}, \mathrm{k}}^{(\mathrm{i})}=\mathrm{B}_{-} \mathrm{W} \log _{2}(1 \\
& \left.+\frac{\mathrm{s}_{\mathrm{j}}^{(\mathrm{i})} \mathrm{P}_{\text {Factor }}^{(\mathrm{i})} \mathrm{P} \rho_{\mathrm{j}, \mathrm{m}, \mathrm{k}}^{(\mathrm{i})}}{1+\sum_{\mathrm{l} \in \mathrm{L} \mathrm{n}}\left(1-\mathrm{P}_{\mathrm{l} \neq \mathrm{j}}^{(\mathrm{i})}{ }_{\text {Factor }}\right) \mathrm{P} \rho_{\mathrm{l}, \mathrm{m}, \mathrm{k}}^{(\mathrm{i})}}\right)
\end{aligned}
$$

\section{Simulation Results}

In this section, simulation results based on Matlab are presented and performed to demonstrate and validate the efficiency of the developed PSA schemes. We assume that the position and the distribution of vehicular users are randomly in the 
coverage area for the current TTI. Simulation results provide a better insight of various issues i.e. average throughput, BER and fairness analysis. In addition, we vary the percentage of ST and NST services, we take as an example three scenarios that are mentioned in table 3 where scenario 1 is oriented Non Safety Traffic (NST), scenario 2 is marked by equity of percentage of traffic and the last one is oriented Safety Traffic (ST), in order to evaluate the system performance and show that our algorithm is efficient and applicable for any scenarios. So the objective of these scenarios is to highlight the effect of the priority on the average blocking rate, the cell throughput and the equity index. Moreover, the performance results of the developed algorithm are compared with the conventional OMA based allocation which is detailed in [29] and some recent works for NOMA based allocation to demonstrate the benefits of our proposed approach.

Table 3: Parameter setting

\begin{tabular}{|c|c|c|c|}
\hline Parameter & \multicolumn{3}{|c|}{ Value } \\
\hline Bandwidth & \multicolumn{3}{|l|}{$10 \mathrm{MHz}$} \\
\hline Available resource blocks & \multicolumn{3}{|l|}{50} \\
\hline $\begin{array}{l}\text { connected VUEs in the } \\
\text { cell }\end{array}$ & \multicolumn{3}{|c|}{$[100,150]$} \\
\hline $\begin{array}{l}\text { eNodeB in the coverage } \\
\text { area }\end{array}$ & \multicolumn{3}{|l|}{1} \\
\hline Transmitted power & \multicolumn{3}{|l|}{$15 \mathrm{dBm}$} \\
\hline $\begin{array}{l}\text { Number of VUEs } \\
\text { allocated the same } \\
\text { spectrum }\end{array}$ & \multicolumn{3}{|l|}{2} \\
\hline TTI & \multicolumn{3}{|l|}{$1 \mathrm{~ms}$} \\
\hline & $\begin{array}{l}\text { Scenario } \\
1\end{array}$ & $\begin{array}{l}\text { Scenario } \\
2\end{array}$ & $\begin{array}{l}\text { Scenario } \\
3\end{array}$ \\
\hline Percentage of ST services & $30 \%$ & $50 \%$ & $70 \%$ \\
\hline $\begin{array}{l}\text { Percentage of MT } \\
\text { services }\end{array}$ & $40 \%$ & $30 \%$ & $20 \%$ \\
\hline Percentage of IT services & $30 \%$ & $20 \%$ & $10 \%$ \\
\hline
\end{tabular}

The used parameters for simulation results are announced below in Table 3.

\subsection{ABR Analysis}

ABR is an important Key Performance Indicators (KPIs) [30]. It is presenting the ratio between the number of blocked transmitter VUEs due to the limited spectrum and the total number of vehicular users defined for each scenario. Moreover, the average blocking rate achieved by the PSA algorithm was calculated by their average values for all scenarios and traced as illustrated in Figure 2.

While increasing the number of connected vehicular UEs in the coverage area, the average blocking rate raises a little or will be perpetually starved in scenario 1 due to the limitation of radio resource blocks. We mark that the raise of average blocking rate is less important for safety traffic in scenario 1 than the others one, those explain that the RBs are assigned based on the type of traffic connections. Scenario 1 is oriented NST traffics, so the number of VST is less than the number of VNST. In addition, the main objective of the proposed approach is to maximize the number of vehiclar users served by assigning a physical RB to two VUEs and by giving more priority to the safety links which must be served immediately due to their timeliness requirement. In scenario 1 and 2 , the user with good channel condition can guarantee the minimum required QoS, has a superior priority to be scheduled while VUE with a mediocre channel gain waiting their access, which conducts to a equity problem and high latency. PSA aims to allocate multiple VUEs with diverse channel qualities simultaneously. Moreover, it can supply an enhancement by ensuring massive connectivity as indicated in Figure 2.

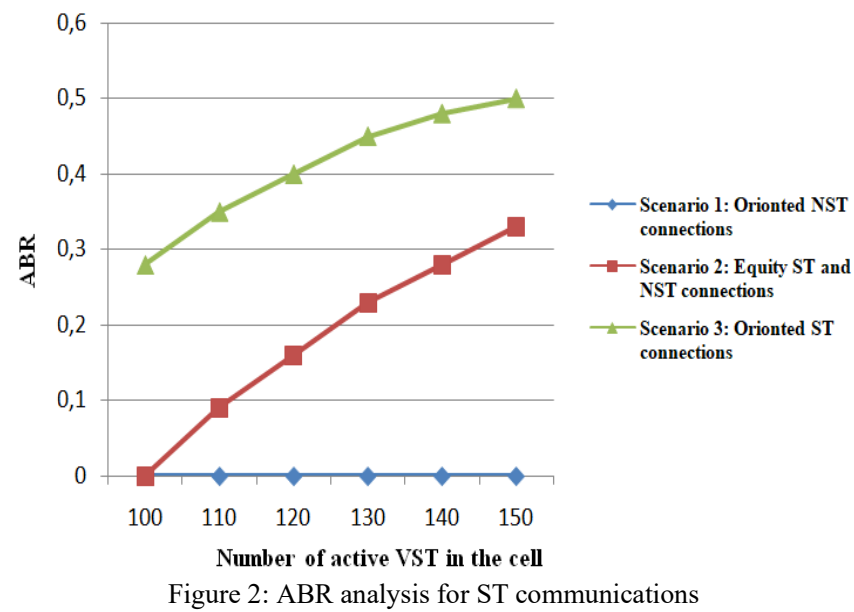

\subsection{Cell Throughput}

Maximizing average throughput is the principal goal of our algorithm. Figure 3 illustrates the performance of PSA scheme in terms of overal throughput for the three already mentioned scenarios versus the number of connected links in the cell.

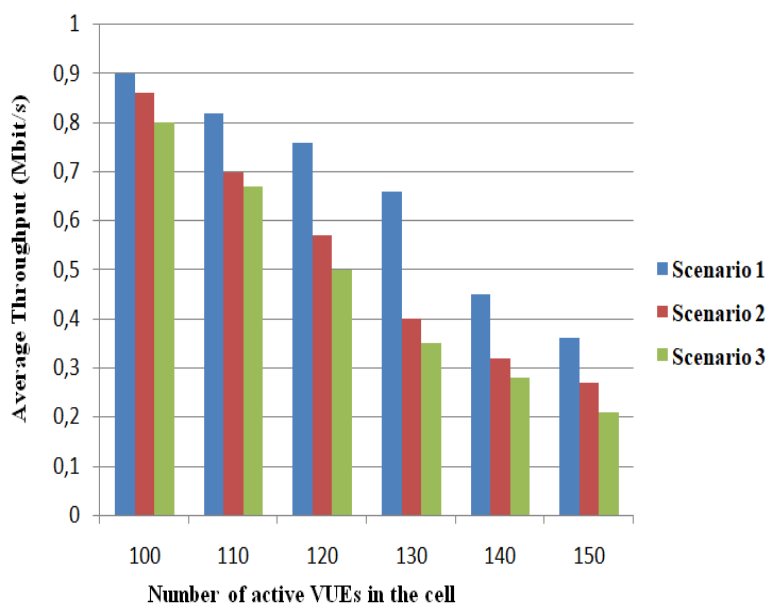

Figure 3: Average throughput analysis for NOMA (Rural Macro-cell)

We show that the raise in cell throughput is more better for scenario 1 than for scenario 2 and scenario 3 which is less efficient. From the Figure 3, it is observed that the total throughput of the proposed algorithm gradually decreases when the number of VUEs increases. This is due to the large demand on resources blocs. Since the proposed algorithm cancels the waste of the RBs by updating the metric before each new allocation and by maximizing the use of available RBs. Indeed, the proposed algorithm achieve 0,9 Mbps (scenario 1) when the targeted active VUEs in the cell is 
equal to 100. This scenario oriented to be NST, while this type of traffics needs a high throughput to provide desired QoS. On the other hand, scenario 3 is oriented safety traffic. Taking into account the type of communications, this increase affects the throughput because in real world scenarios not all V2X connected links are safety communication and not all VUEs are close to the base station. ST connections often requires stringent exigency as will as low latency and high reliability. Indeed, safety traffics representing small data packets compared to non safety traffics. For ST connection like control loss warning, pre-crash sensing warning, forward collision warning and queue warning, the short transmit packets have a size of 50-300 Bytes [31] [32]. Figure 3 shows that the acheived throughput for ST can support several packets sizes. So, PSA allows exchanging packets size along to 120 Bytes when the average sum rate is equal to 0.9 Mbps. Moreover, the results satisfy the recommendation which is imposed by $3 \mathrm{GPP}$ organization when the lowest message size is 50 Bytes.

Indeed, Figure 4 shows how the cell throughput varies according to the number of active connections in the coverage area for the conventional OMA.

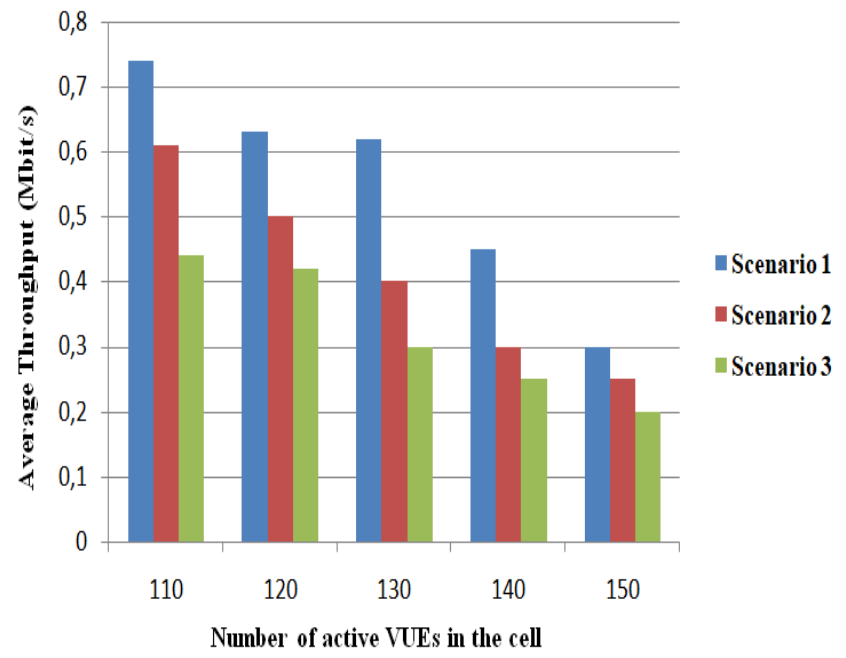

Figure 4: Average throughput analysis for OMA (Rural Macro-cell)

It's obvious that overall throughput decreases when VUEs increases. From that, it is clear that NOMA outperforms OMA even in all scenarios, which verifies that PSA based on NOMA can attain a higher cell throughput than OMA which has a gap to the greatest attainable cell throughput due to it uselessness on active V2X links selection. So, our proposed algorithm out performs OMA-based scheme due to a better resources allocation.

This is because PSA effectively preserves user diversity by its RB allocation metric. Unlike OMA scheduler, NOMA can simultaneously ensure optimal use a huge number of VUEs as well as performing better QoS requirements.

System model of our proposed algorithm does not consider only rural propagation scenario but also urban environment. Figure 5 illustrates the average throughput varies with the number of connected vehicular users in the cell for urban macro-cell propagation scenario evaluated for three different scenarios.

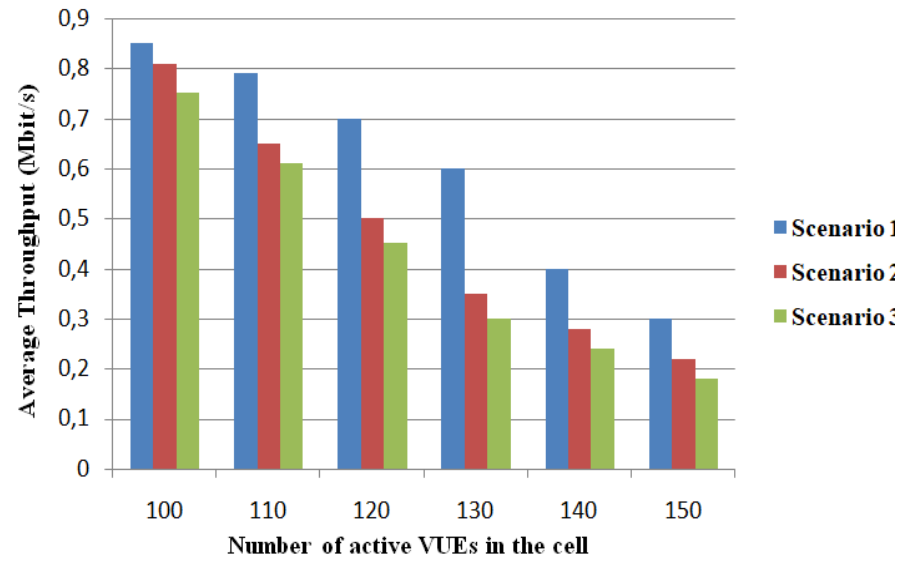

Figure 5: Average throughput analysis (Urban Mcro-cell)

Urban macro-cell propagation will face much path loss due to various properties, such as diffraction, reflection, refraction, absorption, free space loss, coupling loss and aperture-medium compared to the rural macro-cell propagation. This is confirming that the average sum rate for rural macro-cell outperforms the urban macro-cell average throughput as shown in figure 5 . Moreover, all propagation scenarios based on NOMA algorithms are much better compared to OMA one, which confirming the convergence and stability of our proposed algorithm and verifying that NOMA can obtain a higher system throughput than OMA.

In addition, Figure 6 displays the system throughput of the developed resource allocation approach PSA compared with the attainable system throughput in [21] versus of the number of vehicular UEs in the system. Our algorithm is compared to [21], already detailed in related work section, because we find that this reference present the most high throughput compared to other studied related works.

Tree-based Search power Allocation algorithm (TSA) can both solve the link selection problems, user scheduling and power allocation with a low computational complexity for Device to Device underlaying cellular network based on NOMA techniques to increase the average throughput [21].

For FIxed (channel-Independent) Power Allocation algorithm (FIPA), the set of users is sorted in the decreasing normalized channel gain order, the system reaches to assign more power level to users experiencing high channel qualities based on the fixed weighted sum of the instantaneous throughput [33].

For FRactional Power Allocation (FRPA), the transmission power control depends on channel condition variations among users. Respective users must be informed by allocated power through explicit control signaling, which differentiates this allocation from the fixed power allocation [33].

For our proposed PSA algorithm and TSA defined in [21], the cell throughput increases with a varying speed that increasingly slows down due to increasing the number of connected VEUs in the system.

From Figure 6, the increment of the average throughput, PSA is better than TSA and therefore outperforms the other three algorithms FRPA and FIPA. 
Figure 6 also clearly demonstrates the superiority of PSA that outperforms the compared scheme in terms of sum rate since the proposed algorithm takes into account the service classification and the channel gains of the respective non-orthogonally multiplexed VUEs.

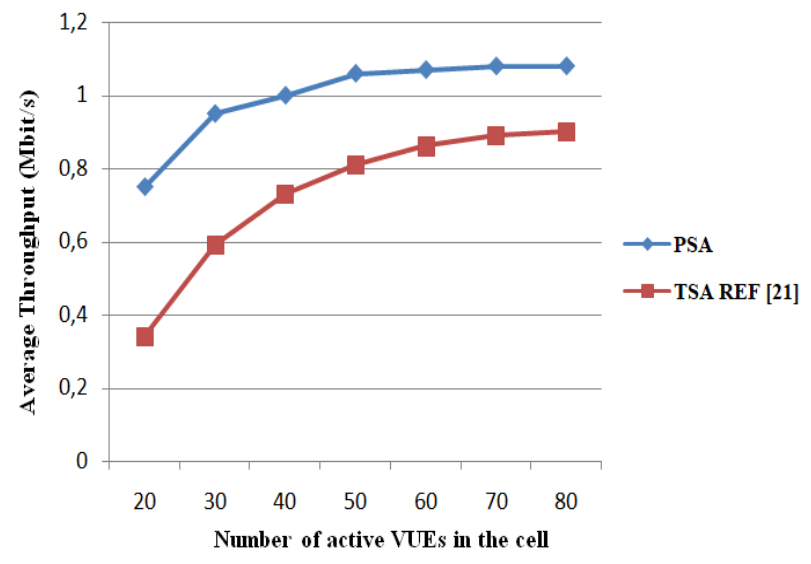

Figure 6: Average throughput analysis

\subsection{Fair Index}

The developed solution focuses on priority management to serve users fairly and increase the number of served VUEs. Fairness is a key factor for evaluating the simulation results of scheduling and resource allocation schemes, its calculates the equity index among VUEs. As PSA aims to maximize the minimum achievable instantaneous data rate, this can ensure fairness among users. To measure the equity of the system, we exploite the well-known techniques, it is Jain's fairness index [34] given by equation "(11)".

$$
f\left(D_{1}, D_{2}, D_{3}, \ldots D_{K}\right)=\frac{\left[\sum_{K=1}^{N_{s}} D_{K}\right]^{2}}{N \sum_{K=1}^{N_{s}}\left(D_{K}\right)^{2}}
$$

where $N_{s}$ is the number of served VST users and $D_{K}$ is the throughput of the Kth VST.

We can observe on Figure 7 that the designed scheme has a good level of equity index for VST because it maximizes the use of the available RB between different V2X communications. Moreover, PSA tend to minimize the rate gap among scheduled users, thus maximizing fairness among them. Comparing with OMA algorithm, the proposed algorithm achieves not only higher system throughput but also better fairness between subscribers.

It was shown that the fairness index of the proposed resource allocation scheme with NOMA system is higher than others paper [18]. The equity of all algorithms decreases when the number of connected links raises. The authors simulate this factor of performance with limited active connections in the system. This demonstrated that the proposed algorithm can ensure the best fairness performance by the different number of connected vehicular users comparing with resultas shown on [18].

Simulation results have illustrate that the proposed algorithm PSA has various proprieties that maintain remarkable enhancement in terms of average throughput, Average Blocking Rate (ABR) and to guarantee fairness index between different class of VUEs comparing to the conventional OMA assisted relay www.astesj.com scheme and some recent work for NOMA based allocation. According to [31] and [32], the simulation results guarantee the $3 \mathrm{GPP}$ recommendation when the minimum small packet size is 50 bytes. Hence, our proposed algorithm is capable to support variable packets sizes meeting the requirements imposed by $3 \mathrm{GPP}$ standard.

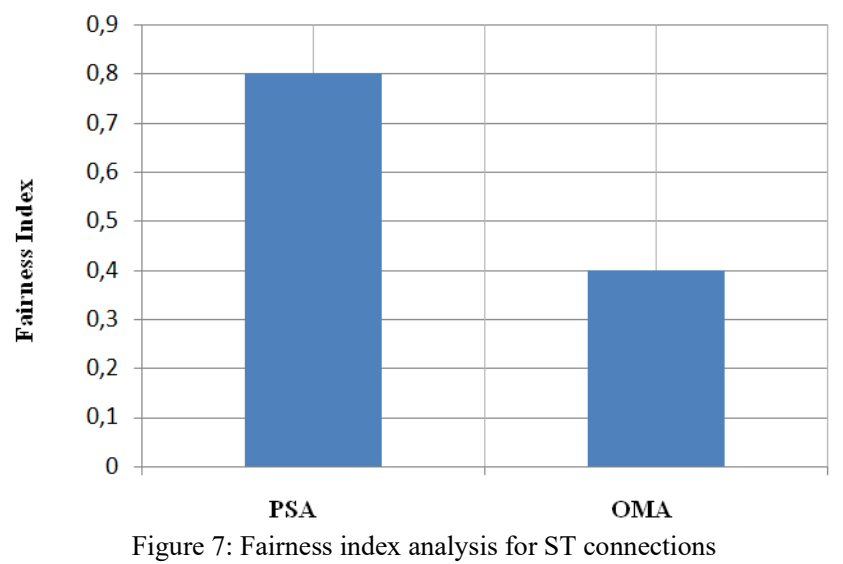

\section{Conclusion}

This paper, presents a novel scheduling and resource allocation approach named PSA for NOMA systems. PSA aims to maintain the highest average throughput by considering the heterogeneity of channels quality of each V2X link. The proposed work increases the number of served safety V2X links in the cell while a minimum requirement must be guaranteed on terms of throughput and maximize the average throughput of NST VUEs. The simulations results verify the optimality of our proposed algorithm in comparison with conventional scheme OMA that lack of caring fairness between vehicular users. The proposed scheme performs amelioration in term of throughput compared to other studied publication papers. A better compromise between cell throughput and fairness index has been achieved. As perspective for this research work, we plan to study the possibilities offered by Quanser platforms for reel environment implementation. In fact Quanser is an open-architecture system integrating plants with realistic dynamics sufficient for physically-relevant testing, realtime computation, and modeling for rapid testing of algorithms and concepts in MATLAB/Simulink and LabVIEW environments. We can also adopt such improvement to optimize the proposed scheme in terms of complexity.

\section{References}

[1] A. Al-Dulaimi, X. Wang, C. Lin, "Machine-Type Communication in the 5G Era: Massive and Ultrareliable Connectivity Forces of Evolution, Revolution, and Complementarity," Wiley-IEEE Press, 2018, doi:10.1002/9781119333142.ch14.

[2] Y. Han et al., "Periodic Radio Resource Allocation to Meet Latency and Reliability Requirements in 5G Networks," IEEE Vehicular Technology Conference (VTC Spring), 2018, doi:10.1109/vtcspring.2018.8417636.

[3] B. Di et al., "Non-Orthogonal Multiple Access for High-Reliable and LowLatency V2X Communications in 5G Systems," IEEE Journal on Selected Areas in Communications, 2017, doi:10.1109/jsac.2017.2726018 .

[4] Z. MacHardy et al., "V2X Access Technologies: Regulation, Research, and Remaining Challenges," IEEE Communications Surveys \& Tutorials, 2018, doi:10.1109/comst.2018.2808444.

[5] A. Masmoudi et al., "A Survey on Radio Resource Allocation for V2X Communication," Wireless Communications and Mobile Computing, 2019, doi:10.1155/2019/2430656. 
[6] F.A. Giovanni, "An Overview of Vehicular Communications," Future Internet, 2019, dio:10.3390/fi11020027.

[7] B. Di et al., "V2X Meets NOMA: Non-Orthogonal Multiple Access for 5GEnabled Vehicular Networks," IEEE Wireless Communications, 2017, doi:10.1109/mwc.2017.1600414.

[8] S. Rai et al., "Result Analysis of Efficient MIMO Framework for NOMA Downlink and Uplink Transmission based on Signal Alignment," International Journal of Computer Applications 2018, doi: 10.5120/ijca2018916907

[9] A. Agarwal et al., "Performance analysis for non-orthogonal multiple access (NOMA)-based two-way relay communication," the Institution of Engineering and Technology (IET Communication), 2019, doi:10.1049/ietcom.2018.5641.

[10] X. He et al., "Spectrum and Power Allocation for Vehicular Networks with Diverse Latency Requirements," IEEE International Conference on Communications, Control, and Computing Technologies for Smart Grids (SmartGridComm), 2019, doi:10.1109/smartgridcomm.2019.8909694.

[11] A. Bazzi et al., "Optimizing the Resource Allocation of Periodic Messages With Different Sizes in LTE-V2V," IEEE Access, 2019, doi:10.1109/access.2019.2908248.

[12] A. Nazari et al., "Resource allocation in power domain NOMA based cooperative multicell networks," the Institution of Engineering and Technology (IET Communication), 2020, doi: 10.1049/iet-com.2019.0624.

[13] N. Glei et al., "Power Allocation for Energy-Efficient Downlink NOMA Systems," international conference on Sciences and Techniques of Automatic control \& computer engineering (STA), 2019, doi: $10.1109 /$ STA.2019.8717240.

[14] K. Huang et al., "Energy efficient resource allocation algorithm in multicarrier NOMA systems," International Conference on High Performance Switching and Routing (HPSR), 2019, doi:10.1109/HPSR.2019.8807992.

[15] Z. Yuan "Energy Efficient Power Allocation for Multi-carrier Nonorthogonal Multiple Access (NOMA) Systems with Proportional Rate Constraints," IEEE 3rd Information Technology, Networking, Electronic and Automation Control Conference (ITNEC), 2019, doi:10.1109/ITNEC.2019.8729390.

[16] M. Zeng et al., "Spectral- and Energy-Efficient Resource Allocation for Multi-Carrier Uplink NOMA Systems," IEEE Transactions on Vehicular Technology, 2019, doi:10.1109/TVT.2019.2926701.

[17] M. Pischella et al., "NOMA-Relevant Clustering and Resource Allocation for Proportional Fair Uplink Communications," IEEE Wireless Communications Letters, 2019, doi:10.1109/lwc.2019.2897796.

[18] K. Long et al., "Spectrum Resource and Power Allocation With Adaptive Proportional Fair User Pairing for NOMA Systems," IEEE Access, 2019, doi:10.1109/access.2019.2908673.

[19] X. Wang et al., "Low-Complexity Power Allocation in NOMA Systems With Imperfect SIC for Maximizing Weighted Sum-Rate," IEEE Access, 2019, doi:10.1109/access.2019.2926757.

[20] R. Abozariba et al., "NOMA based Resource Allocation and Mobility Enhancement Framework for IoT in Next Generation Cellular Networks," IEEE Access, 2019, doi:10.1109/access.2019.2896008.

[21] J. Wang et al., "A Novel Resource Allocation Scheme in NOMA-Based Cellular Network with D2D Communications," Future Internet, 2020, doi: $10.3390 /$ fi1 12010008 .

[22] L. Chen et al., "Proportional Fairness-Based User Pairing and Power Allocation Algorithm for Non-Orthogonal Multiple Access System," IEEE Access, 2019, doi:10.1109/access.2019.289618.

[23] H. Xing et al., "Sum-rate Maximization Guaranteeing User Fairness for NOMA in Fading Channels," IEEE Wireless Communications and Networking Conference (WCNC), 2018, doi:10.1109/wcnc.2018.8376949.

[24] J. He et al., "Design and Optimization of Scheduling and Non-orthogonal Multiple Access Algorithms with Imperfect Channel State Information," IEEE Transactions Vehicular Technology, 2018, doi:10.1109/tvt.2018.2869305.

[25] METIS D1.4, METIS Channel Models, ICT-317669-METIS/D1.4, METIS project, 2013.

[26] WINNER II D1.1.2, Channel models, V1.2, 2008.

[27] G. Dimitrakopoulos et al., "Vehicular Communications Standards, Current Technologies in Vehicular Communication. Springer, Cham, 2017.

[28] C.M. Silva et al., "A Survey on Infrastructure-Based Vehicular Networks," Hindawi Mobile Information Systems, 2017, doi:10.1155/2017/6123868.

[29] A.D. Trabelsi et al., "Dynamic Scheduling Algorithm based on Priority Assignment for LTE-V2X vehicular Networks," International Wireless Communications \& Mobile Computing Conference (IWCMC), 2019, doi:10.1109/iwcmc.2019.8766632.

[30] G. Cecchini et al., "Localization-Based Resource Selection Schemes for www.astesj.com
Network-Controlled LTE-V2V," International Symposium on Wireless Communication Systems (ISWCS), 2017, doi: 10.1109/ISWCS.2017.8108147.

[31] 3rd Generation Partnership Project, Technical Specification Group Services and System Aspects: Study on LTE support for Vehicle to Everything (V2X) services(Release 14), 3GPP TR 22.885 V14.0.0, 2015.

[32] 3rd Generation Partnership Project, Technical Specification Group Services and System Aspects; Study on enhancement of 3GPP Support for 5G V2X Services (Release 15), 3GPP TR 22.886 V15.1.0, 2017.

[33] N. Otao et al., "Performance of Non-orthogonal Access with SIC in Cellular Downlink Using Proportional Fair-Based Resource Allocation," International Symposium on Wireless Communication Systems (ISWCS), 2012, doi:10.1109/iswcs.2012.6328413.

[34] R. Jain, The Art of Computer Systems Performance Analysis, Wiley, 1991. 